EPJ Web of Conferences 60, 17010 (2013)

DOI: $10.1051 /$ epjconf $/ 20136017010$

(C) Owned by the authors, published by EDP Sciences, 2013

\title{
Searches for New Physics in Top-like Final States
}

\author{
Yeng-Ming Tzeng ${ }^{1, a}$, on behalf of the CMS Collaboration \\ ${ }^{1}$ National Taiwan University
}

\begin{abstract}
We present searches for massive top and bottom quark partners at CMS using data collected at $\sqrt{s}=7$ and $8 \mathrm{TeV}$. Such partners are incorporated in 4th generation models, or can be found in other models, able to solve the Hierarchy problem and stabilize the Higgs mass. Other searches presented here focus on excited states of composite heavy objects that decay to top quarks and jets. The searches span a range of final states, from multi-leptonic to entirely hadronic, and limits are set on mass and production cross sections as a function of branching ratios.
\end{abstract}

\section{Introduction}

A new scalar boson of mass of about $125 \mathrm{GeV} / c^{2}[1]$ has been discovered in the LHC era.

Even though this new scalar boson could be the ultimate particle predicted by the standard model (SM), an additional mechanism is needed in order to solve the hierarchy problem produced by the quadratically divergent loop contributions to the Higgs mass (see Figure 1).

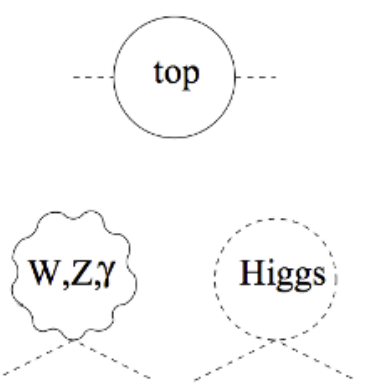

Figure 1. The quadratically divergent contributions to the Higgs mass in the standard model, from top quark, gauge bosons, and Higgs itself.

A number of new models are proposed to solve the hierarchy problem. For instance, the little Higgs models [2] introduce a vector-like top (T) quark and a $\mathrm{W}^{\prime}$ boson to cancel the divergencies from the top loop and the $\mathrm{W}$ loop, respectively. In cerntain vector-like models [3], whose left- and right-handed fermions transform in the same way under the $\mathrm{SM}$ gauge groups $\mathrm{SU}(3)_{C} \times \mathrm{SU}(2)_{L} \times \mathrm{U}(1)_{Y}$, a triplet vector-like quark is composed of a top partner with a charge of $5 / 3\left(\mathrm{~T}_{5 / 3}\right)$, a vector-like top quark $(\mathrm{T})$, and a vector-like bottom quark (B). These new fundamental particles could decay to final states including a top quark, such as in the decays $\mathrm{T} \rightarrow \mathrm{tZ}, \mathrm{W}^{\prime+} \rightarrow \mathrm{t} \overline{\mathrm{b}}, \mathrm{T}_{5 / 3} \rightarrow \mathrm{tW}^{+}$, and $\mathrm{B} \rightarrow \mathrm{tW}^{-}$.

Futhermore, the inner structure of a top quark is worth investigating. The composite top quark can be directly tested by a search for an excited top quark $\left(\mathrm{t}^{*}\right)$, which mostly decays into a top quark and a gluon [10].

\footnotetext{
a e-mail: ymtzeng@cern.ch
}

Searches for new particles in final states including top quarks are extremely motivated, and presented in this report.

\section{Searches for New Particles}

The searches presented in this paper can be classified in two categories, according to the investigated final states : the top + jet and top $+\mathrm{W}$ final states. Each of the mentioned categories can be split into two sub-categories.

In the first category, if the jet is coming from the hadronization of $\mathrm{a} b$ quark, the new particle searched for could be regarded as a $\mathrm{W}^{\prime}$ boson. According to a previous CMS [4] result using an integrated luminosity of 5 $\mathrm{fb}^{-1}$ of $7 \mathrm{TeV}$ collision data, a $\mathrm{W}^{\prime}$ boson with right-handed couplings to fermions with masses below $1.85 \mathrm{TeV}$ [5] is excluded at $95 \%$ C.L.. If the jet originates from a gluon, the decay could be associated to an excited top quark $\left(\mathrm{t}^{*}\right)$. In the second category, two sub-categories can be considered according to the W-boson's charge. A top partner $\left(\mathrm{T}_{5 / 3}\right)$ can decay into a top quark and a $\mathrm{W}$ boson of positive charge. For this decay channel a mass limit is set by CMS, using $7 \mathrm{TeV}$ collision data, up to $645 \mathrm{GeV}$ [6]. If the $\mathrm{W}$ boson in the final state has a negative charge, the decay could be from a vector-like bottom quark (B), of which the mass is already excluded up to $675 \mathrm{GeV}$ [7].

Table 1. A summary table for exclusion limits on the masses of resonances having a top-like final state with $7 \mathrm{TeV}$ collision data.

\begin{tabular}{l|ll}
\hline Final state & Model & Lower mass limit \\
\hline \hline \multirow{2}{*}{ top+jet } & $\mathrm{W}^{\prime} \rightarrow \mathrm{tb}$ & $1.85 \mathrm{TeV}[5]$ \\
& $\mathrm{t}^{*} \rightarrow \mathrm{tg}$ & Not probed yet. \\
\hline \multirow{2}{*}{ top+W } & $\mathrm{T}_{5 / 3} \rightarrow \mathrm{tW}$ & $645 \mathrm{GeV}[6]$ \\
& $\mathrm{B} \rightarrow \mathrm{tW}$ & $675 \mathrm{GeV}[7]$ \\
\hline
\end{tabular}


These measurements using $7 \mathrm{TeV}$ collision data are summarized in Table 1. The latest results with $8 \mathrm{TeV}$ collision data will be presented in the following sub-sections.

\subsection{Top + b Quark Resonance}

The search for singly produced $\mathrm{W}^{\prime}$ in the tb channel, is performed using a leptonic final state [8]. A $\mathrm{W}^{\prime}$ boson with an arbitrary combination of left- and right-handed couplings to fermions is assumed as signal. The event selection involves one isolated lepton (electron or muon), $\geq$ 2 jets, and $\geq 1$ b-tagged jet.

The invariant mass of top $+b$-jet system, being the combination of the reconstructed top and the highest $p_{\mathrm{T}}$ jet which is not associated with the top quark is displayed in Figure 2 and 3. In addition the reconstructed top is obtained using the combination of the charged lepton, the neutrino, and the jet, which gives the best top-quark mass.

In order to reduce the SM background constribution, additional selections, including a top mass window, the top's $p_{\mathrm{T}}$, and the sum of two leading jets' $p_{\mathrm{T}}$, are applied.

The most dominant background contributions to be modeled are the $\bar{t}+$ jets and $\mathrm{W}+$ jets processes. The $\mathrm{W}+$ jets shape is obtained without considering the b-tag requirement. The $t \bar{t}+$ jets shape is given by applying a ratio function from a top's $p_{\mathrm{T}}$ fit.

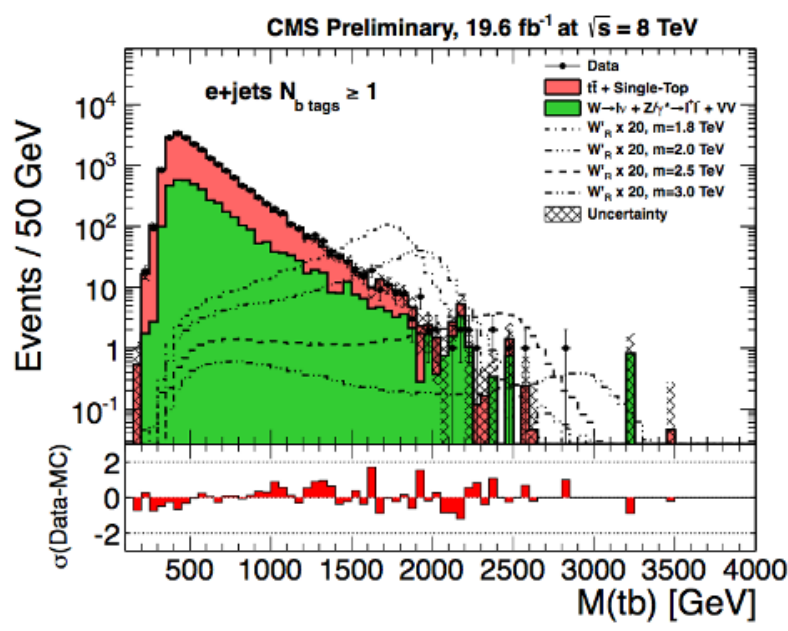

Figure 2. The invariant mass of $\mathrm{W}^{\prime}$ boson in the electron channel reconstructed after the full selection. For the purpose of illustration, the expected signal yields are scaled by a factor of 20 .

For $\mathrm{W}^{\prime}$ bosons with right-handed couplings to fermions, we exclude masses below $2.03 \mathrm{TeV}$ at $95 \%$ C.L. (see Figure 4). The limits on $\mathrm{W}^{\prime}$ masses for arbitrary leftand right-handed couplings are presented in Figure 5.

\subsection{Top + Gluon Resonance}

The search for a pair-production of $t^{*}$ decaying to a top and a gluon in the semi-leptonic channel [9], is presented in this sub-section. The event selection is of one isolated lepton (electron or muon), $\geq 6$ jets, and $\geq 1$ b-tagged jet.

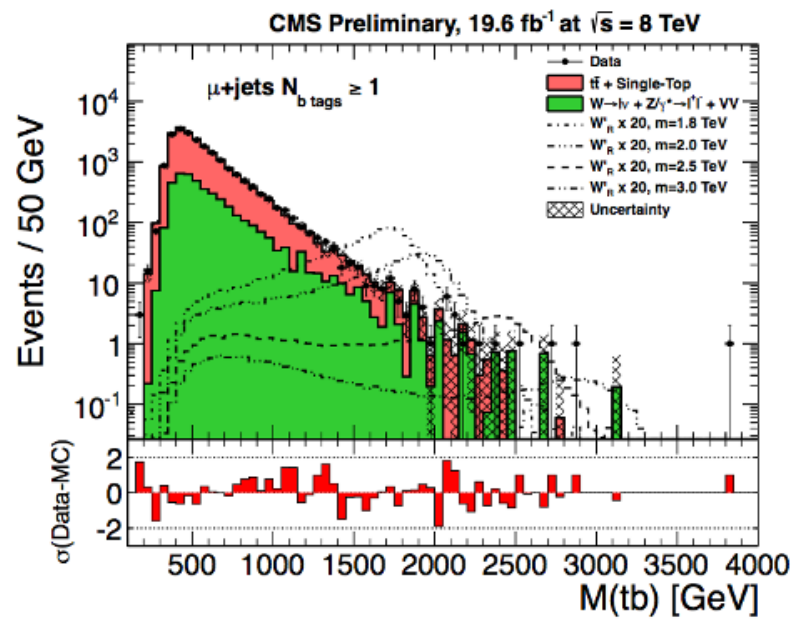

Figure 3. The invariant mass of $\mathrm{W}^{\prime}$ boson in the muon channel reconstructed after the full selection. For the purpose of illustration, the expected signal yields are scaled by a factor of 20 .

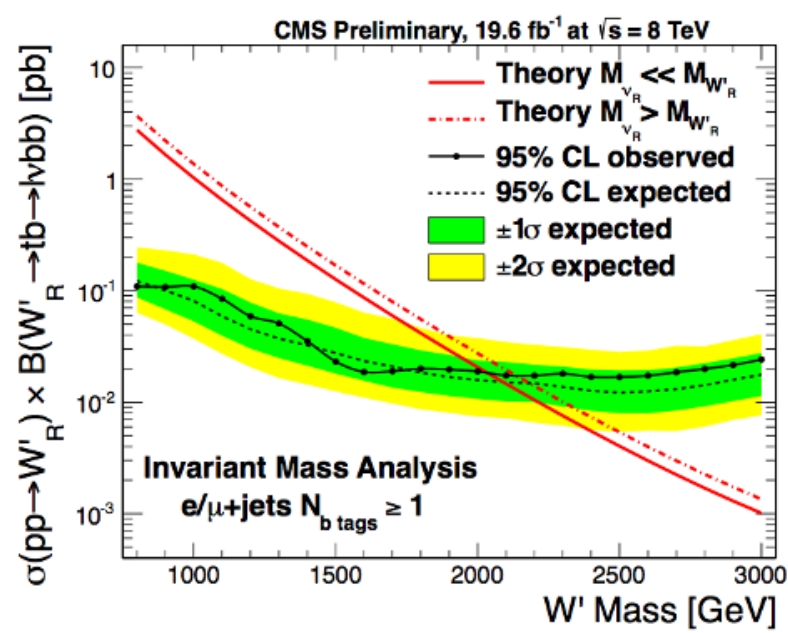

Figure 4. The expected and observed 95\% C.L. upper limits on the production cross-section of right-handed $\mathrm{W}^{\prime}$ bosons obtained for the combination of the electron and muon channels.

The invariant mass of top+jet system in Figure 6 and 7 is reconstructed using a kinematic fit.

The SM background shape is modeled by using a fermi-like function, $b g(m)=\frac{a}{1+e^{(m-b) / c}}$. A global fit to data is performed, while the signal model from simulation is used.

This result is interpreted in terms of a spin-3/2 top quark partner [10]. Under the assumption of $100 \%$ branching fraction of $\mathrm{t}^{*} \rightarrow \operatorname{tg}$, excited top quarks $\left(\mathrm{t}^{*}\right)$ with masses less than $794 \mathrm{GeV}$ are excluded at $95 \%$ C.L. (see Figure 8).

\subsection{Top + W Resonance with Charge 5/3}

The search for a pair-production of $\mathrm{T}_{5 / 3}$ which decays into a top quark and a $\mathrm{W}$ boson, is performed in the same-sign dilepton channel [11]. The Cambridge-Aachen (CA)-based substructure algorithm is used to reconstruct 


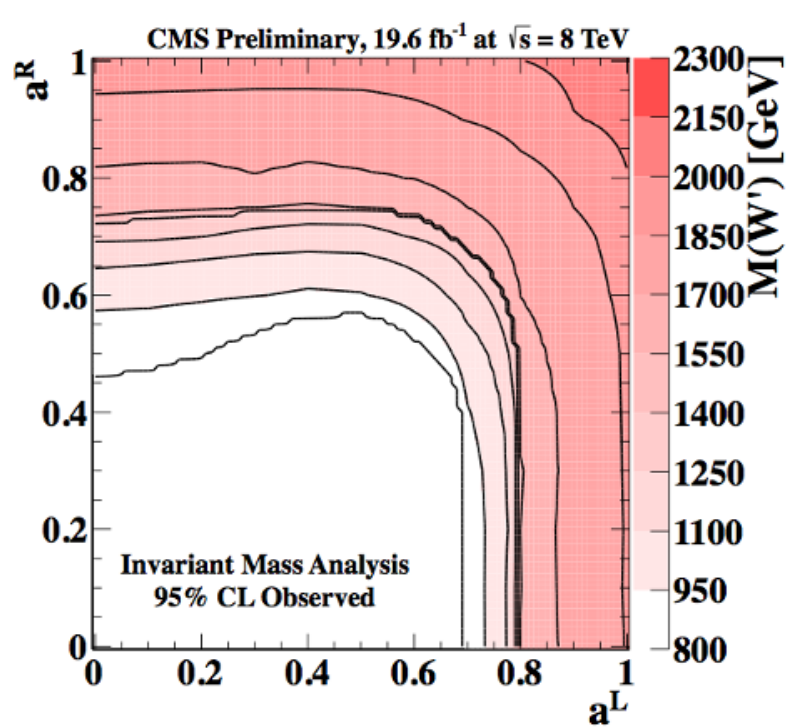

Figure 5. Contour plot for observed $95 \%$ C.L. limits on $\mathrm{M}\left(\mathrm{W}^{\prime}\right)$ with an arbitrary combination of left-handed $\left(\mathrm{a}^{\mathrm{L}}\right)$ and righthanded $\left(\mathrm{a}^{\mathrm{R}}\right)$ couplings.

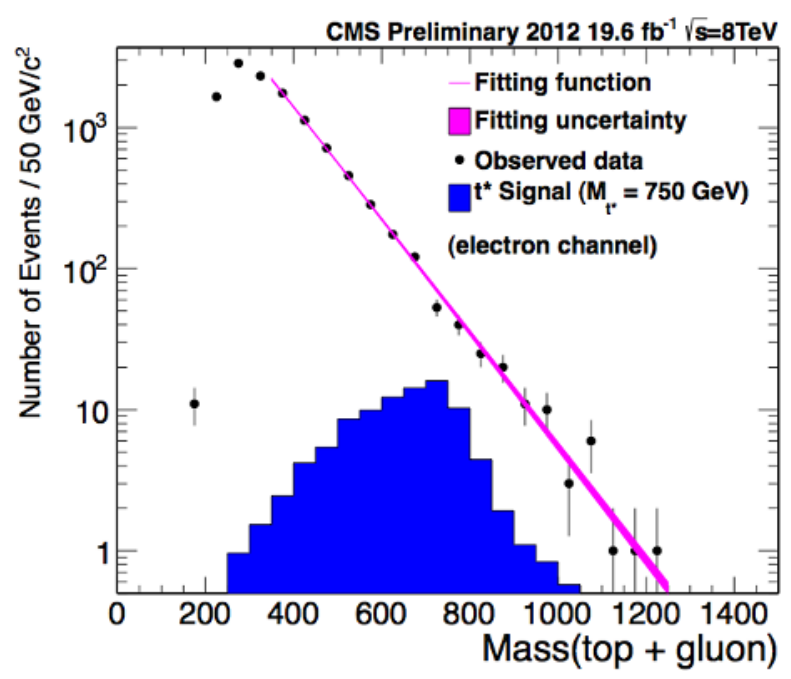

Figure 6. The top + gluon invariant mass reconstructed in the electron channel using a kinematic fit with the smallest $\chi^{2}$ value.

a boosted top quark or a W boson [12]. Event selection includes two isolated leptons with same-sign charge, quarkonia veto, $\mathrm{Z}$ boson veto, and at least 5 subjets and additional jets reconstructed with the Anti- $\mathrm{k}_{\mathrm{T}}$ algorithm of radius 0.5 (Ak5) [13].

The invariant mass of a top quark and a $\mathrm{W}$ boson (see Figure 9) is reconstructed by taking into account four kinds of conditions. In general, a reconstructed top quark and a reconstructed $\mathrm{W}$ boson are needed, and the reconstruction method depends on different conditions. If CA top jets and $\mathrm{CA} \mathrm{W}$ jets both exist, the reconstructed mass is given by the combination of CA top and CA W jets. If only CA top jets exist, a reconstructed $\mathrm{W}$ boson will be perfomed using Ak5 jets. If only $\mathrm{CA} \mathrm{W}$ jets exist, a top quark will be reconstructed using Ak5 jets. Otherwise,

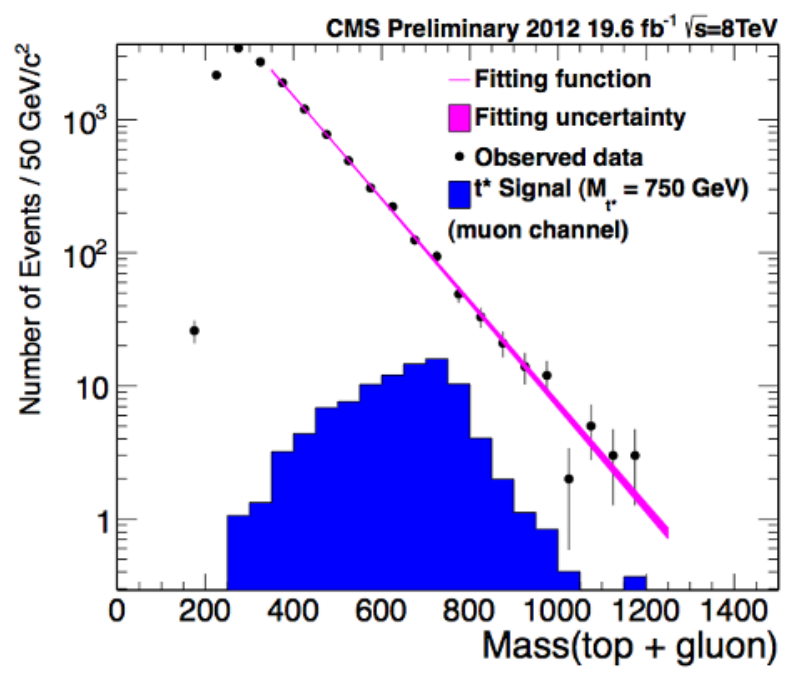

Figure 7. The invariant mass of the top + gluon system in the muon channel reconstructed using a kinematic fit with the smallest $\chi^{2}$ value.

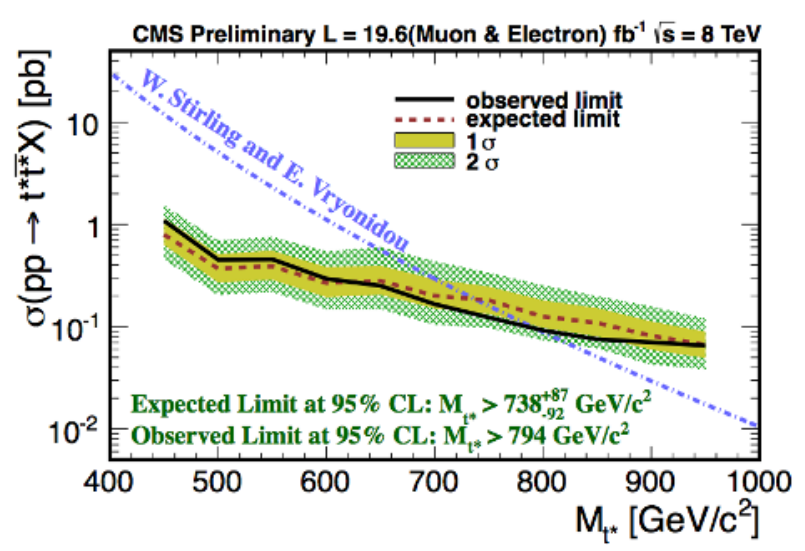

Figure 8. The expected and observed 95\% C.L. upper limits for the $\mathrm{t}^{*} \mathrm{t}^{*}$ production cross section as a function of the $\mathrm{t}^{*}$ mass for the combination of the channels.

the top quark and the $\mathrm{W}$ boson will be reconstructed using Ak5 jets.

The estimated backgrounds are from three types of sources. Of the first type are the processes with samesign prompt dilepton (WW, WZ, ZZ, ttW/Z, and WWW), which are obtained using simulated samples. Of the second type are events with opposite-sign prompt dilepton, and a charge mis-identified rate measured from a Z-control sample is used to estimate this contribution. The last type is composed by events with same-sign non-prompt dilepton from a heavy-flavor decay, decay in flight, or conversion. Also in this case, the fake rate is measured in data.

An exclusion limit (see Figure 10) is set for masses below $770 \mathrm{GeV}$ at $95 \%$ C.L., when assuming $100 \%$ branching fraction of $\mathrm{T}_{5 / 3} \rightarrow \mathrm{tW}$. 


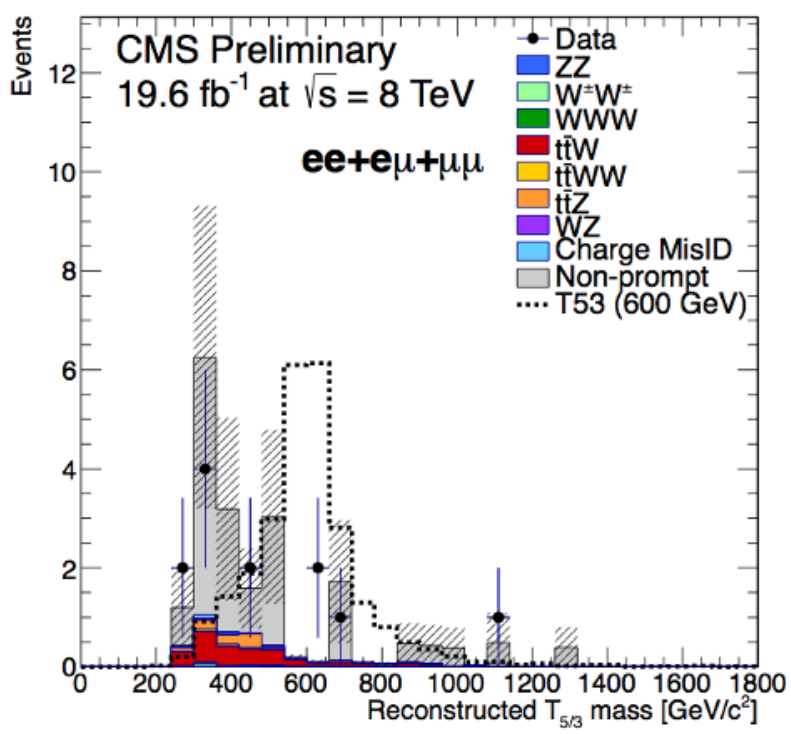

Figure 9. Reconstructed $T_{5 / 3}$ mass for the combination of all three dilepton channels after requiring the same-sign dilepton and $\mathrm{Z}$ /quarkonia lepton veto.

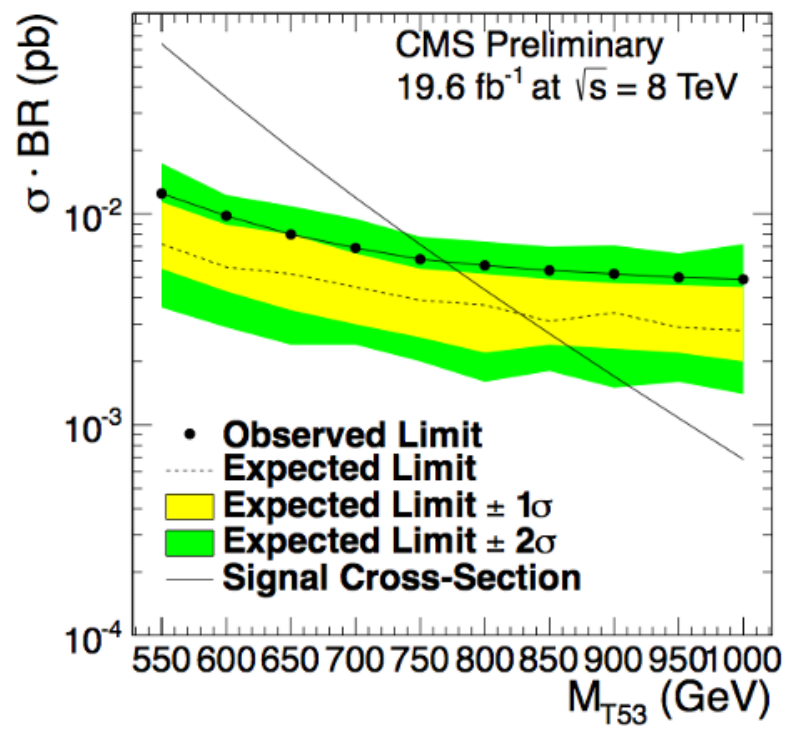

Figure 10. Expected and observed limits at $95 \%$ C.L. on the $\mathrm{T}_{5 / 3}$ production cross section. Statistical and systematic uncertainties are included.

\subsection{Top + W Resonance with Charge $1 / 3$}

The search for a pair-production of $b^{\prime}$ which decays into a top quark and a $\mathrm{W}$ boson is performed in the multi-lepton channel [14]. Every event is selected with requirements of 3 (4) isolated leptons, 1 (2) opposite-sign same-flavor (OSSF) lepton pair (pairs), one OSSF pair outside (inside) the $\mathrm{Z}$ mass window, and at least one b-tagged jet.

The sum of objects' $p_{\mathrm{T}}$ (see Figure 11), defined as $\mathrm{S}_{\mathrm{T}}=\mathrm{MET}+\sum$ (lepton's $\left.p_{\mathrm{T}}\right)+\sum\left(\right.$ jet's $\left._{\mathrm{T}}\right)$, is used as main observable for this search.

Data-driven methods take into account background contributions from misidentified/non-prompt leptons and asymmetric photon conversions. Simulated samples are used to estimate contributions from irreducible backgrounds, including $\mathrm{t} \overline{\mathrm{t}}$, diboson, and $\mathrm{t} \mathrm{t} W / \mathrm{Z}$.

For $\mathrm{BR}\left(\mathrm{b}^{\prime} \rightarrow \mathrm{tW}\right)=100 \%$, masses below $760 \mathrm{GeV}$ are excluded at $95 \%$ C.L. (see Figure 12). The contribution from $\mathrm{b}^{\prime} \rightarrow \mathrm{bZ}$ is also taken into account by defining $\mathrm{BR}\left(\mathrm{b}^{\prime} \rightarrow \mathrm{tW}\right)=1-\mathrm{BR}\left(\mathrm{b}^{\prime} \rightarrow \mathrm{bZ}\right)$. Limits range between 660 and $760 \mathrm{GeV}$ as the braching fraction is varied (see Figure 13).

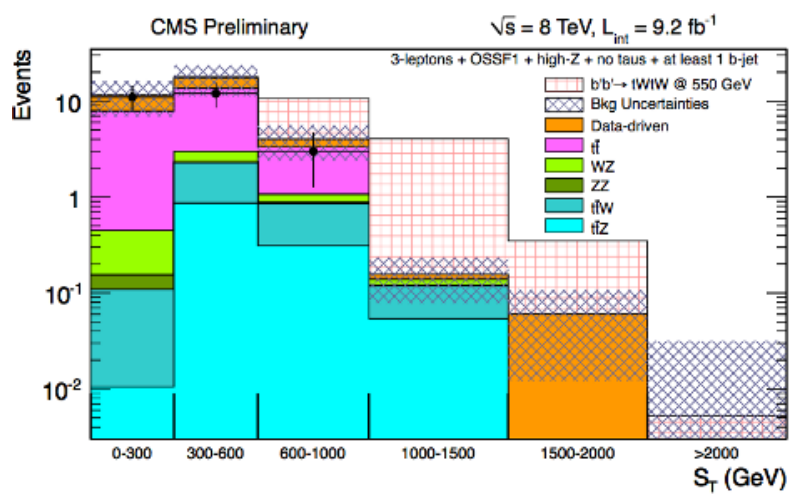

Figure 11. $S_{\mathrm{T}}$ distribution for the three-lepton channel with at least one b-tagged jet and Z-vetoed OSSF pair of leptons, assuming $\mathrm{b}^{\prime} \rightarrow \mathrm{tW}$ with $100 \%$ branching fraction.

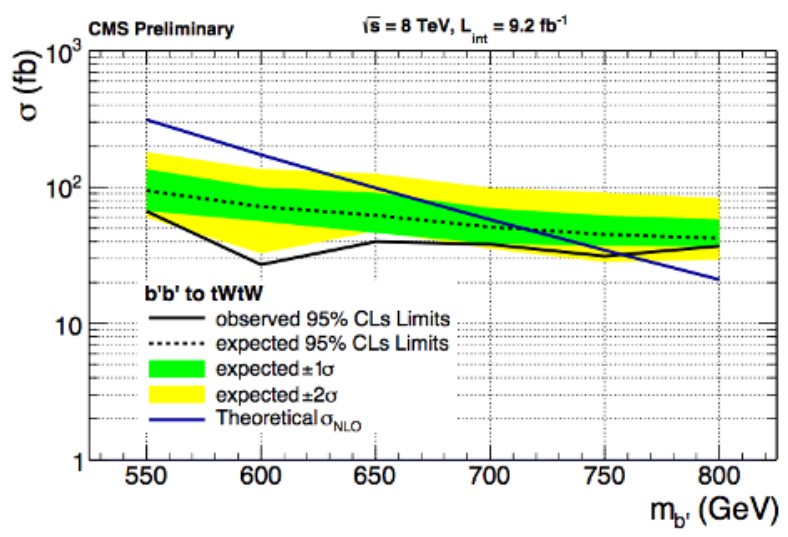

Figure 12. The one-dimensional exclusion limit plot, assuming $100 \%$ branching fraction of $b^{\prime} \rightarrow t W$.

\section{Summary}

The CMS collaboration has performed a number of searches for new physics in top-like final states with $8 \mathrm{TeV}$ collision data. Up to now, no evidence for new physics has been found. Exclusion limits on the masses of resonances having a final state characterized by the presence of top quark are summarized in Table 2.

\section{References}

[1] CMS Collaboration, Observation of a new boson at a mass of $125 \mathrm{GeV}$ with the CMS experiment at the LHC, Physics Letters B 716, 30-61 (2012) 


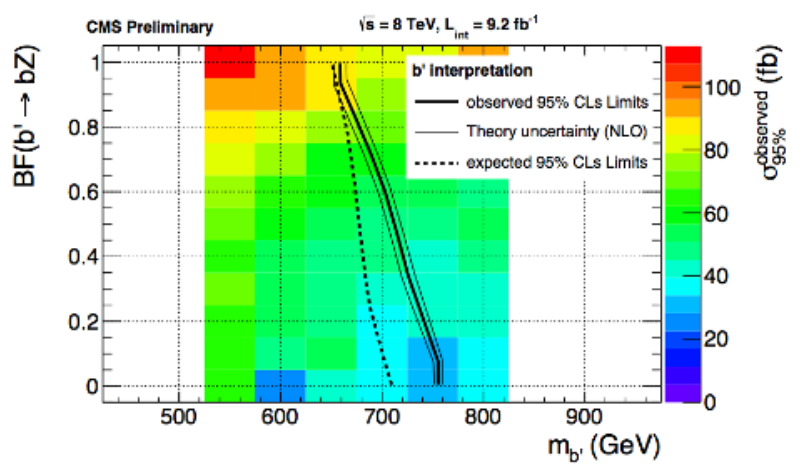

Figure 13. Exclusion limits in the two-dimensional plane, $\mathrm{BR}\left(\mathrm{b}^{\prime} \rightarrow \mathrm{bZ}\right)$ versus $\mathrm{b}^{\prime}$ mass, where $\mathrm{BR}\left(\mathrm{b}^{\prime} \rightarrow \mathrm{tW}\right)$ is regarded as $1-\mathrm{BR}\left(\mathrm{b}^{\prime} \rightarrow \mathrm{bZ}\right)$.

Table 2. Summary table of exclusion limits on the masses of resonances having a final state containing a top quark with 8 $\mathrm{TeV}$ collision data.

\begin{tabular}{l|ll}
\hline Final state & Model & Lower mass limit \\
\hline \hline \multirow{2}{*}{ top+jet } & $\mathrm{W}^{\prime} \rightarrow \mathrm{tb}$ & $2.03 \mathrm{TeV}[8]$ \\
& $\mathrm{t}^{*} \rightarrow \mathrm{tg}$ & $794 \mathrm{GeV}[9]$ \\
\hline \multirow{2}{*}{ top+W } & $\mathrm{T}_{5 / 3} \rightarrow \mathrm{tW}$ & $770 \mathrm{GeV}[11]$ \\
& $\mathrm{b}^{\prime} \rightarrow \mathrm{tW}$ & $760 \mathrm{GeV}[14]$ \\
\hline
\end{tabular}

[2] Martin Schmaltz, Introducing the Little Higgs, Nucl.Phys.Proc.Suppl. 117, 40-49 (2003)

[3] Cacciapaglia, Giacomo and Deandrea, Aldo and Harada, Daisuke and Okada, Yasuhiro, Bounds and Decays of New Heavy Vector-like Top Partners, JHEP 1011, 159 (2010)

[4] CMS Collaboration, The CMS experiment at the CERN LHC, JINST 3, S08004 (2008)
[5] CMS Collaboration, Search for Heavy $W^{\prime}$ Boson resonances decaying to a Bottom Quark and a Top Quark at $\sqrt{s}=7 \mathrm{TeV}$, CMS Physics Analysis Summary CMSPAS-EXO-12-001 (2012)

[6] CMS Collaboration, Search for a heavy partner of the top quark with charge 5/3, CMS Physics Analysis Summary CMS-PAS-B2G-12-003 (2012)

[7] CMS Collaboration, Search for $b^{\prime}$ pair production in the lepton + jets channel, CMS Physics Analysis Summary CMS-PAS-B2G-12-004 (2012)

[8] CMS Collaboration, Search for $t+b$ resonances in leptonic final state, CMS Physics Analysis Summary CMS-PAS-B2G-12-010 (2012)

[9] CMS Collaboration, Search for pair production of new physics resonances decaying to a top quark and jet in the lepton+jets decay channel, CMS Physics Analysis Summary CMS-PAS-B2G-12-014 (2012)

[10] Hassanain, Babiker and March-Russell, John and Rosa, J.G., On the possibility of light string resonances at the LHC and Tevatron from Randall-Sundrum throats, JHEP 0907, 077 (2009)

[11] CMS Collaboration, Search for T5/3 top partners in same-sign di-lepton final state, CMS Physics Analysis Summary CMS-PAS-B2G-12-012 (2012)

[12] CMS Collaboration, Jet Substructure Algorithms, CMS Physics Analysis Summary CMS-PAS-JME-10013 (2010)

[13] M. Cacciari, G. P. Salam and G. Soyez, The anti-kt jet clustering algorithm, JHEP 0804, 063 (2008).

[14] CMS Collaboration, Search for RPV supersymmetry with three or more leptons and b-tags, CMS Physics Analysis Summary CMS-PAS-SUS-12-027 (2012) 